\title{
Risk Analysis for Latent Infection of Prune by Monilinia fructicola in California
}

\author{
Yong Luo and Themis J. Michailides
}

Department of Plant Pathology, University of California-Davis, Kearney Agricultural Center, Parlier, CA 93648. Accepted for publication 1 September 2001.

\begin{abstract}
Luo, Y., and Michailides, T. J. 2001. Risk analysis for latent infection of prune by Monilinia fructicola in California. Phytopathology 91:1197-1208.

The quantitative relationships between incidence of latent infection (ILI) of prune by Monilinia fructicola and wetness duration (WD) for different bloom and fruit developmental stages and different inoculum concentrations were obtained. Three levels of ILI were considered as criteria for low, moderate, and high risks of latent infection, respectively. Seasonal patterns of WD leading to different risk levels of latent infection were obtained for low $\left(\mathrm{IP}_{L}\right)$ and high $\left(\mathrm{IP}_{H}\right)$ inoculum potential conditions in orchards. Longer WD was needed at a resistant than at a susceptible fruit developmental stage to induce similar levels of latent infection. The curves of WD leading to different levels of ILI over the growing season (risky WD curves) were used in risk analysis for latent infection. Multi-year historical WD data from 10 prune-growing loca-
\end{abstract}

ABSTRACT

In California, statewide epidemics of brown rot of stone fruit caused by Monilinia fructicola (G. Wint.) Honey, occur infrequently. However, orchard- and region-scale epidemics causing different yield losses frequently occur depending on many factors in orchards such as inoculum potential (IP), weather condition, and cultural practice in orchards (16-19). Two phases of this disease should be considered in decision making for disease management, blossom blight and fruit brown rot. Blossom blight is caused by ascospores or conidia produced from mummified fruit that were infected by $M$. fructicola, and it usually happens in early spring $(4,9-11,24)$. Severe blossom blight may cause flower rot and reduce the number of fruit. The susceptibility of prune blossoms to infection by $M$. fructicola over different developmental stages, the relationships between disease incidence and environments, as well as the possible risk of blossom blight associated with different environments were intensively studied (14).

Under favorable conditions, infections could continue on prune fruit after bloom. Infection of fruit is usually caused by conidia produced on infected blossoms, mummies, or thinned fruit and could cause serious yield losses $(6,9,25)$. Secondary infections may occur during the growing season when favorable weather conditions are encountered. Many of these infections can be latent for a period until they become active brown rot symptoms on fruit with sporulation. Studies on inoculum sources showed that nonabscised, aborted fruit in trees and thinned fruit on orchard floors infected by $M$. fructicola could serve as secondary inoculum, causing fruit brown rot $(1,10,18)$. The significance of thinned fruit as a source of secondary inoculum in nectarine orchards in California was also confirmed (8). Inoculum potential in a specific

Corresponding authors: Y. Luo and T. Michailides

E-mail addresses: yluo@uckac.edu and themis@uckac.edu

Publication no. P-2001-1016-02R

(C) 2001 The American Phytopathological Society tions in California were compared with risky WD curves. The percentage of days $(\mathrm{P})$ with WD leading to a certain risk level of latent infection was calculated for each month from historical weather data. Under the $\mathrm{IP}_{L}$ condition, the $\mathrm{P}$ distributions for low risk of latent infection were higher in March and April than in May and were the lowest in June for most locations. Under the $\mathrm{IP}_{H}$ condition, the number of days that WD leading to low risk of latent infection in May increased compared with those under the $\mathrm{IP}_{L}$ condition. The risk analysis approach was evaluated by using separate experimental data as incidence of fruit brown rot obtained from different prune orchards over years. Consistency between predicted overall risk levels of latent infection and observed incidence of fruit brown rot was obtained. The results demonstrated the usefulness of the risk analysis in decision support system for disease management.

Additional keywords: Prunus domestica, stone fruit. prune orchard is dependent on disease history, orchard environment, cultural practices, and disease control strategies. In prune orchards in California, disease symptoms and sporulation usually do not appear until late in the season when higher humidity and near optimum temperatures are common.

Our previous studies (13) in three prune orchards determined the seasonal pattern of bloom and fruit susceptibility to latent infection by $M$. fructicola. The susceptibility to latent infection at bloom stage was at a moderate level, increased to reach to the highest level at about pit hardening stage, subsequently, decreased, reaching the lowest level in early June at embryo growth, and increased again along with fruit development and maturity until harvest. The incidence of latent infection (ILI) increased exponentially with increased wetness duration (WD) and linearly with increased inoculum concentration (IC) for most bloom and fruit developmental stages (13). These findings confirmed similar studies that used detached peach fruit $(2,7)$.

These studies (13) also implied that the same or similar WD and IP may lead to different latent infection levels at different fruit developmental stages. Therefore, environmental criteria used for disease control might be different throughout the growing season. For example, a certain WD, such as $12 \mathrm{~h}$, might bring about a high risk of latent infection early in the season but a low risk in the middle of the season. Understanding the seasonal patterns of environmental conditions leading to different risk levels of latent infection is therefore important in decision making for disease management.

In California, the commercial recommendation of fungicide spray to control blossom blight is at the green tip stage. Orchards with different levels of IP may have different possibilities of latent infection, especially in those orchards where the disease infrequently occurred. However, the importance in reducing latent infection after bloom is still underestimated. Our pervious study (13) found that the stage of the highest susceptibility to latent infection was from fruit set to embryo growth, implying that the 
risk of latent infection causing severe disease is not only at the bloom stage. The microclimatic conditions in orchards after bloom are critical in disease appearance in the season, and an estimation of risk of disease development throughout the growing season becomes critical in disease management.

WD, IC, and temperature all affect latent infection of prune by M. fructicola $(12,13)$. Similar findings were reported on other stone fruits $(3,5,18,20,22)$. How the risk of latent infection changes at different fruit developmental stages and what environments could affect this change need to be addressed for the development of a decision support system for disease control. This study emphasized WD and IC as critical factors for development of a risk analysis approach. The objectives of this study were to (i) determine the relationships between ILI and WD for different bloom and fruit developmental stages, (ii) determine the seasonal pat- terns of WD leading to different risk levels of latent infection, (iii) analyze historical weather patterns to estimate the occurrence of the weather leading to risks of latent infection for different areas in California, and (iv) evaluate the risk analysis approach by comparing predicted risk levels of latent infection with observed incidences of fruit brown rot.

\section{MATERIALS AND METHODS}

Seasonal pattern of bloom and fruit susceptibility to latent infection. In a previous study, experiments were conducted in three prune orchards located in Yolo, Butte, and Tehama counties in California. In each orchard, trees were inoculated at full bloom and four fruit developmental stages: pit hardening, embryo growth, late embryo growth, and before first harvest (23). Five
Step1:
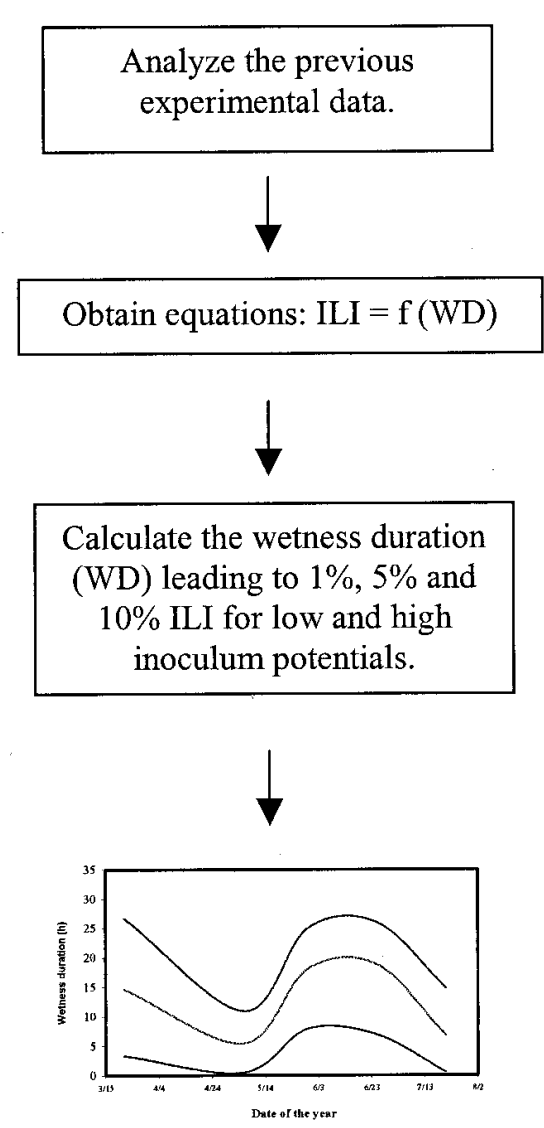

Obtain curves of wetness duration leading to different risk levels of latent infection over the growing season.
Step 2:

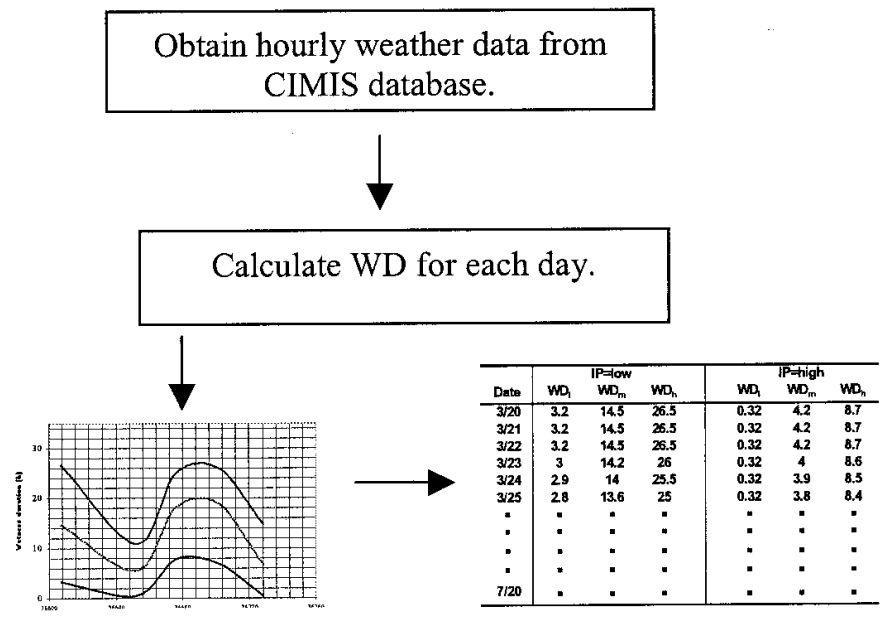

Determine the risky WD for each day using the results of step 1, and develop a table containing daily WD leading to different risk levels of latent infection.
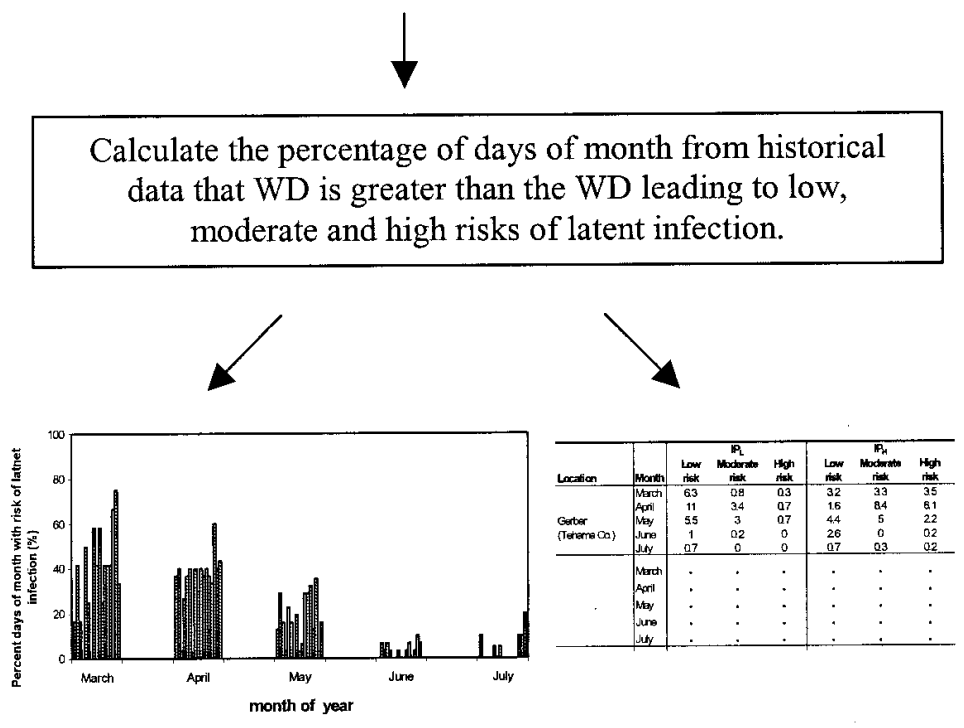

Obtain the distributions in percent days of the month with WD leading to a certain risk level of latent infection (P), and develop a table containing the average number of days (threshold ND) with WD leading to different risk levels of latent infection.

Fig. 1. A schematic diagram showing approaches of risk analysis for latent infection of prune by Monilinia fructicola in which two steps were used. Step 1 obtained the quantitative relationships between incidence of latent infection (ILI) and wetness duration (WD) and determined the seasonal patterns of WD leading to different levels of latent infection. Step 2 used the results of step 1 to analyze the probability of occurrence of latent infection for different locations in California using historical weather data. 
ICs, $1,000,5,000,10,000,20,000$, and 30,000 conidia per $\mathrm{ml}$ of M. fructicola, were used in inoculation on the branches, each bearing 40 to 60 flowers or fruits by spraying. The inoculated branches were covered with plastic bags to maintain high humidity. Six WDs $(4,8,12,16,20$, and $24 \mathrm{~h})$ for bloom and four WDs $(4,8,12$, and $16 \mathrm{~h})$ for fruit developmental stages were generated for each inoculation by uncovering plastic bags at different times. Fruit on all inoculated branches were maintained on trees until harvest and were processed by an overnight freezing incubation technique $(16,17)$ to determine the ILI. A seasonal pattern of bloom and fruit susceptibility to latent infection by M. fructicola was obtained. A moderate level of ILI occurred at bloom. The fruit susceptibility to infection increased after bloom stage, and the maximum ILI was at about pit hardening stage. Subsequently, susceptibility of fruit to latent infection decreased, reaching the lowest level in early June at the embryo growth stage, and increased again along with fruit development and maturity until harvest. The ILI increased exponentially with increased WD in most treatments at ambient orchard temperatures (13).

Analysis of experimental data. In order to obtain and simplify the relationships between ILI and WD for bloom and fruit developmental stages, the ICs used in the previous study were classified as low, moderate, and high IC. Data of ILI for 1,000 conidia per $\mathrm{ml}$ were used for the low IC. Data of ILI for 5,000 and 10,000 conidia per $\mathrm{ml}$ were combined for the moderate IC, and data of ILI for 20,000 and 30,000 conidia per ml were combined for the high IC. An exponential regression, ILI $=a+b \times e^{c \times \mathrm{WD}}$, was applied to determine the relationships between ILI and WD for each bloom and fruit developmental stage and for each of the three IC levels using the computer software SigmaPlot (version 5.0, SPSS Inc., Richmond, CA). Because no correlation between ILI and WD was found for IC $=1,000$ conidia per $\mathrm{ml}$ at bloom and two fruit stages, this IC was omitted in the following analyses. Subsequently in this study, the moderate IC refers to a low inoculum potential $\left(\mathrm{IP}_{L}\right)$ and the high $\mathrm{IC}$, the high inoculum potential $\left(\mathrm{IP}_{H}\right)$, representing two field conditions.

For each $\mathrm{IP}_{L}$ and $\mathrm{IP}_{H}$ condition, four risk levels of latent infection were assigned as follows: no risk when ILI $<1 \%$, low risk when $1 \% \leq \mathrm{ILI}<5 \%$, moderate risk when $5 \% \leq \mathrm{ILI}<10 \%$, and high risk when ILI $\geq 10 \%$.

The WDs leading to the corresponding risk levels were calculated by converting the above exponential regressions as: WD = $\ln [(\mathrm{ILI}-a) / b] / c$, where $a, b$, and $c$ are parameters of the regression. Thus, the $\mathrm{WD}_{1}, \mathrm{WD}_{5}$, and $\mathrm{WD}_{10}$ were calculated by using the above formula as ILI $=1,5$, and $10 \%$, respectively. These three values were referred to as the critical WD leading to low, moderate, and high risk of latent infection, respectively, and were determined for each bloom and fruit developmental stage under $\mathrm{IP}_{L}$ and $\mathrm{IP}_{H}$ conditions calculated by the corresponding regressions.

Approaches of risk analysis for latent infection. Two steps were involved in the risk analysis approaches (Fig. 1). Step 1 used the regressions obtained from the above analysis to determine the seasonal patterns of WD leading to different risk levels of latent infection. Step 2 analyzed the historical weather data for prunegrowing locations in California to obtain the frequency distributions of WD leading to different risk levels of latent infection for each location.

The $\mathrm{WD}_{1}, \mathrm{WD}_{5}$, and $\mathrm{WD}_{10}$ values for each developmental stage were separately used to draw the curves over the growing season from 15 March to 20 July for $\mathrm{IP}_{L}$ and $\mathrm{IP}_{H}$ conditions using the computer software Microsoft Excel (Microsoft Corp., Bothell, WA). Therefore, three curves representing the WDs leading to 1 , 5 , and $10 \%$ of ILI, respectively, over the growing season were obtained. The corresponding WD values on the curves are referred to as the risky WD, and the curves are referred to as the risky WD curves. In addition to this study, these curves can also be used as a reference to estimate risk levels of latent infection over the growing season (Fig. 1).
In order to estimate possible risk of latent infections in California, historical weather data from 1983 to 2000, including hourly air temperature, dew point temperature (degree Celsius), and precipitation (millimeter) for 10 prune-growing locations, were collected from the California Irrigation Management Information System. These locations ranged from central San Joaquin Valley to northern Sacramento Valley (Fig. 2). For each hour, wetness was determined by comparing the air temperature and dew point temperature and by checking the precipitation using a switch variable, $D$, as follows: $D=1$, when air temperature is less than dew point temperature or precipitation is greater than $0, D=0$, when air temperature is greater than dew point temperature and precipitation equals 0.

WD (hours) was calculated by summarizing $D$ for each day. This calculation was confirmed by comparing the above calculated WD with the leaf wetness measurement using a data logger (Onset Computer Corporation, Bourne, MA) with a leaf wetness sensor for 3 weeks. The calculated daily WDs were equaled to those of the measurement during the 3 weeks.

From the risky WD curves described previously, the $\mathrm{WD}_{1}$, $\mathrm{WD}_{5}$, and $\mathrm{WD}_{10}$ values for each day from 15 March through 20 July were determined on a scaled sheet (Fig. 1) and were used to develop a table. This table contained seven columns: date, the corresponding daily $\mathrm{WD}_{1}, \mathrm{WD}_{5}$, and $\mathrm{WD}_{10}$ values for the $\mathrm{IP}_{L}$ condition, and the corresponding $\mathrm{WD}_{1}, \mathrm{WD}_{5}$, and $\mathrm{WD}_{10}$ values for the $\mathrm{IP}_{H}$ condition (Fig. 1). This table was compare with the historical wetness duration $\left(\mathrm{WD}_{\text {his }}\right)$ for each day. The variables $\mathrm{RK}_{L}, \mathrm{RK}_{M}$, and $\mathrm{RK}_{H}$ were used to count the number of historical days associated with low, moderate, and high risk of latent infection, respectively, for both $\mathrm{IP}_{L}$ and $\mathrm{IP}_{H}$ conditions: $\mathrm{RK}_{L}=1$ when $\mathrm{WD}_{1} \leq$ $\mathrm{WD}_{\text {his }}<\mathrm{WD}_{5}$, otherwise $\mathrm{RK}_{L}=0 ; \mathrm{RK}_{M}=1$ when $\mathrm{WD}_{5} \leq \mathrm{WD}_{\text {his }}<$ $\mathrm{WD}_{10}$, otherwise $\mathrm{RK}_{M}=0$; and $\mathrm{RK}_{H}=1$ when $\mathrm{WD}_{\text {his }} \geq \mathrm{WD}_{10}$, otherwise $\mathrm{RK}_{H}=0$. Where $\mathrm{RK}_{L}, \mathrm{RK}_{M}$, and $\mathrm{RK}_{H}$ are assigned as 0 or 1 to determine if the $\mathrm{WD}_{\text {his }}$ leading to low, moderate, and high risk of latent infection, respectively, exists.

The $\mathrm{RK}_{L}, \mathrm{RK}_{M}$, and $\mathrm{RK}_{H}$ were separately summarized for each month of each year to obtain the number of days with WD leading to low $\left(\mathrm{ND}_{L}\right)$, moderate $\left(\mathrm{ND}_{M}\right)$, and high $\left(\mathrm{ND}_{H}\right)$ risks of latent infection, respectively. The percentage of days in the month with low risk $\left(\mathrm{P}_{L}\right)$, moderate risk $\left(\mathrm{P}_{M}\right)$, and high risk $\left(\mathrm{P}_{H}\right)$ of latent infection, respectively, were also calculated by dividing $\mathrm{ND}_{L}$,

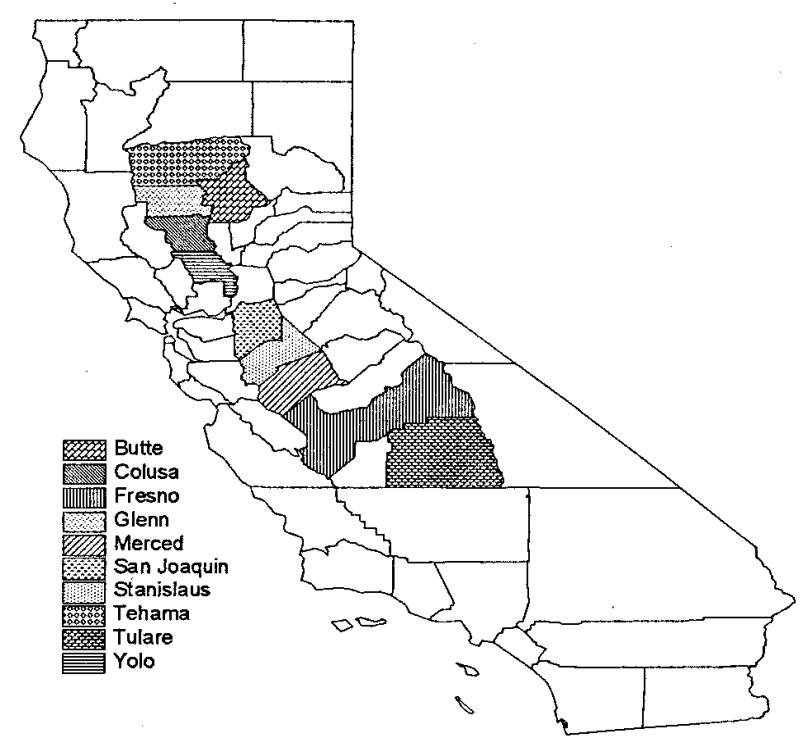

Fig. 2. Risk analysis for latent infection of prune by Monilinia fructicola was conducted for 10 prune-growing counties in California. Historical weather data were collected and wetness durations leading to different risk levels of latent infection were generated for each of these counties as references for disease management. 
$\mathrm{ND}_{M}$, and $\mathrm{ND}_{H}$ by the total number of days in the month, respectively. These ND values are referred to as the threshold ND values. The yearly $\mathrm{P}_{L}, \mathrm{P}_{M}$, and $\mathrm{P}_{H}$ data were used to obtain their distributions for each month and each location (Fig. 1). The monthly mean $\mathrm{P}_{L}, \mathrm{P}_{M}$, and $\mathrm{P}_{H}$ and the $\mathrm{P}_{L}, \mathrm{P}_{M}$, and $\mathrm{P}_{H}$ values with $90 \%$ cumulative probability, $\mathrm{CP}_{L 90}, \mathrm{CP}_{M 90}$, and $\mathrm{CP}_{H 90}$, respectively, were calculated for each month. The monthly means of threshold $\mathrm{ND}_{L}, \mathrm{ND}_{M}$, and $\mathrm{ND}_{H}$ for each location under each IP condition were used as references of historical conditions associated with different risks of latent infection (Fig. 1). All calculations involved in the risk analysis approaches were performed by SAS (version 8.0, SAS Institute, Cary, NC).

Evaluation of risk analysis approaches. The previously observed fruit brown rot data were used to evaluate the predictability of the above risk analysis approaches. Two evaluations were conducted with two sets of experimental data. Evaluation 1 used the

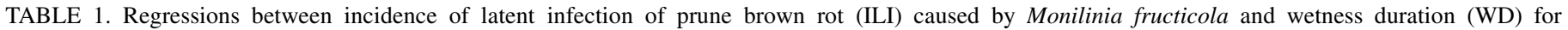
combinations of the bloom and fruit developmental stages and inoculum concentrations

\begin{tabular}{|c|c|c|c|c|c|}
\hline Developmental stage & $\begin{array}{l}\text { Inoculum concentration } \\
\qquad(\text { conidia } / \mathrm{ml})\end{array}$ & Equation & $n$ & $r$ & $P$ \\
\hline \multirow[t]{4}{*}{ Full bloom } & 1,000 & Not correlated & 32 & $\cdots$ & $\cdots$ \\
\hline & $5,000-10,000$ & $\mathrm{ILI}=-23.77+23.7123 \exp (0.0132 \mathrm{WD})^{\mathrm{a}}$ & 70 & 0.43 & 0.0012 \\
\hline & $20,000-30,000$ & $\mathrm{ILI}=-181.95+182.61 \exp (0.0057 \mathrm{WD})$ & 59 & 0.60 & 0.0002 \\
\hline & 1,000 & $\mathrm{ILI}=0.22+\exp (0.2084 \mathrm{WD})$ & 67 & 0.28 & 0.0204 \\
\hline \multirow[t]{3}{*}{ Pit hardening } & $5,000-10,000$ & $\mathrm{ILI}=-0.2335+2.4556 \exp (0.1391 \mathrm{WD})$ & 127 & 0.41 & $<0.0001$ \\
\hline & $20,000-30,000$ & $\mathrm{ILI}=7.2683+0.3203 \exp (0.3165 \mathrm{WD})$ & 134 & 0.75 & $<0.0001$ \\
\hline & 1,000 & Not correlated & 60 & $\ldots$ & $\ldots$ \\
\hline \multirow[t]{2}{*}{ Embryo growth } & $5,000-10,000$ & $\mathrm{ILI}=-2.3517+2.0219 \exp (0.0712 \mathrm{WD})$ & 134 & 0.38 & $<0.0001$ \\
\hline & $20,000-30,000$ & $\mathrm{ILI}=-0.8302+1.5062 \exp (0.0601 \mathrm{WD})$ & 126 & 0.22 & 0.0418 \\
\hline \multirow[t]{3}{*}{ Late embryo growth } & 1,000 & $\mathrm{ILI}=-0.1708+0.0818 \exp (0.3349 \mathrm{WD})$ & 41 & 0.62 & 0.0001 \\
\hline & $5,000-10,000$ & $\mathrm{ILI}=-1.7275+1.6015 \exp (0.0763 \mathrm{WD})$ & 82 & 0.42 & 0.0006 \\
\hline & $20,000-30,000$ & $\mathrm{ILI}=-1.2518+0.7362 \exp (0.2365 \mathrm{WD})$ & 102 & 0.58 & $<0.0001$ \\
\hline \multirow[t]{3}{*}{ Before first harvest } & 1,000 & Not correlated & 67 & $\ldots$ & $\ldots$ \\
\hline & $5,000-10,000$ & $\mathrm{ILI}=-3.7178+6.0093 \exp (0.0556 \mathrm{WD})$ & 129 & 0.37 & 0.0001 \\
\hline & $20,000-30,000$ & $\mathrm{ILI}=-1.5789+5.0619 \exp (0.1060 \mathrm{WD})$ & 126 & 0.51 & $<0.0001$ \\
\hline
\end{tabular}

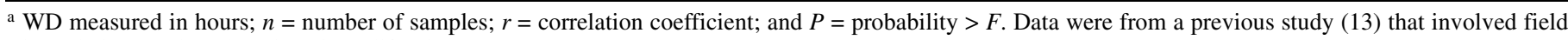
inoculations at three locations in California.

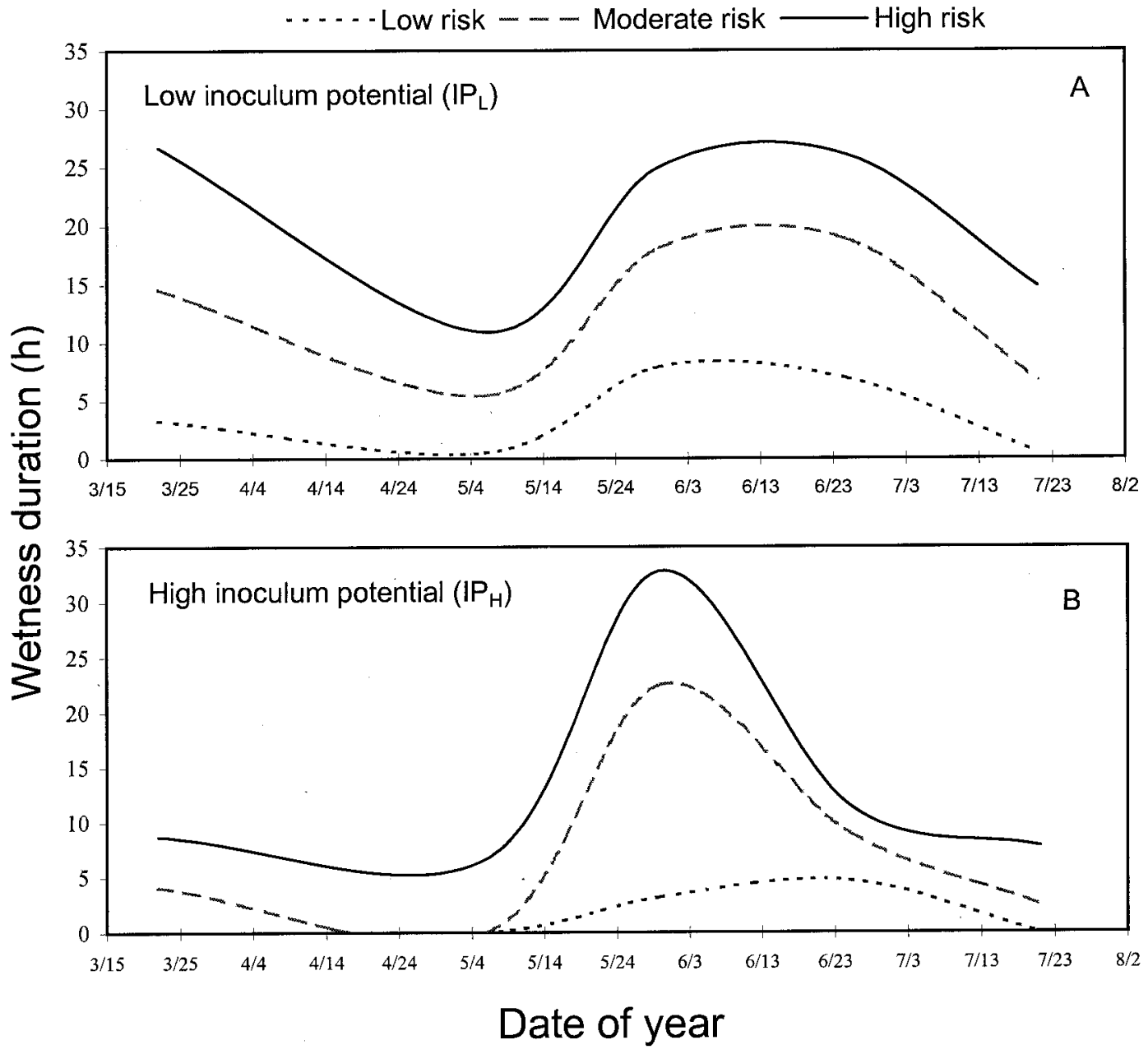

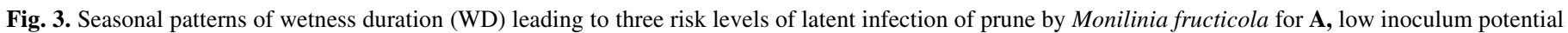
and $\mathbf{B}$, high inoculum potential conditions. The curves were developed based on the quantitative relationships between incidence of latent infection and WD. 
experimental data obtained from prune orchards in four counties (Tulare, Tehama, Butte, and Sutter) in California in 1993 (20). The objective of the experiments was to determine the effects of early and midsummer sprays of fungicides on control of prune brown rot at harvest and postharvest. The average incidence of fruit brown rot from noninoculated (naturally infected) trees, the non- treatment control, was used in the evaluation process. The IP in the orchard in Tulare County was estimated as low $\left(\mathrm{IP}_{L}\right)$, because severe brown rot infrequently occurred in the orchard compared with those located in northern California. The IPs in the remaining three orchards were high $\left(\mathrm{IP}_{H}\right)$. The corresponding daily WDs leading to low, moderate, and high risks of latent infection were
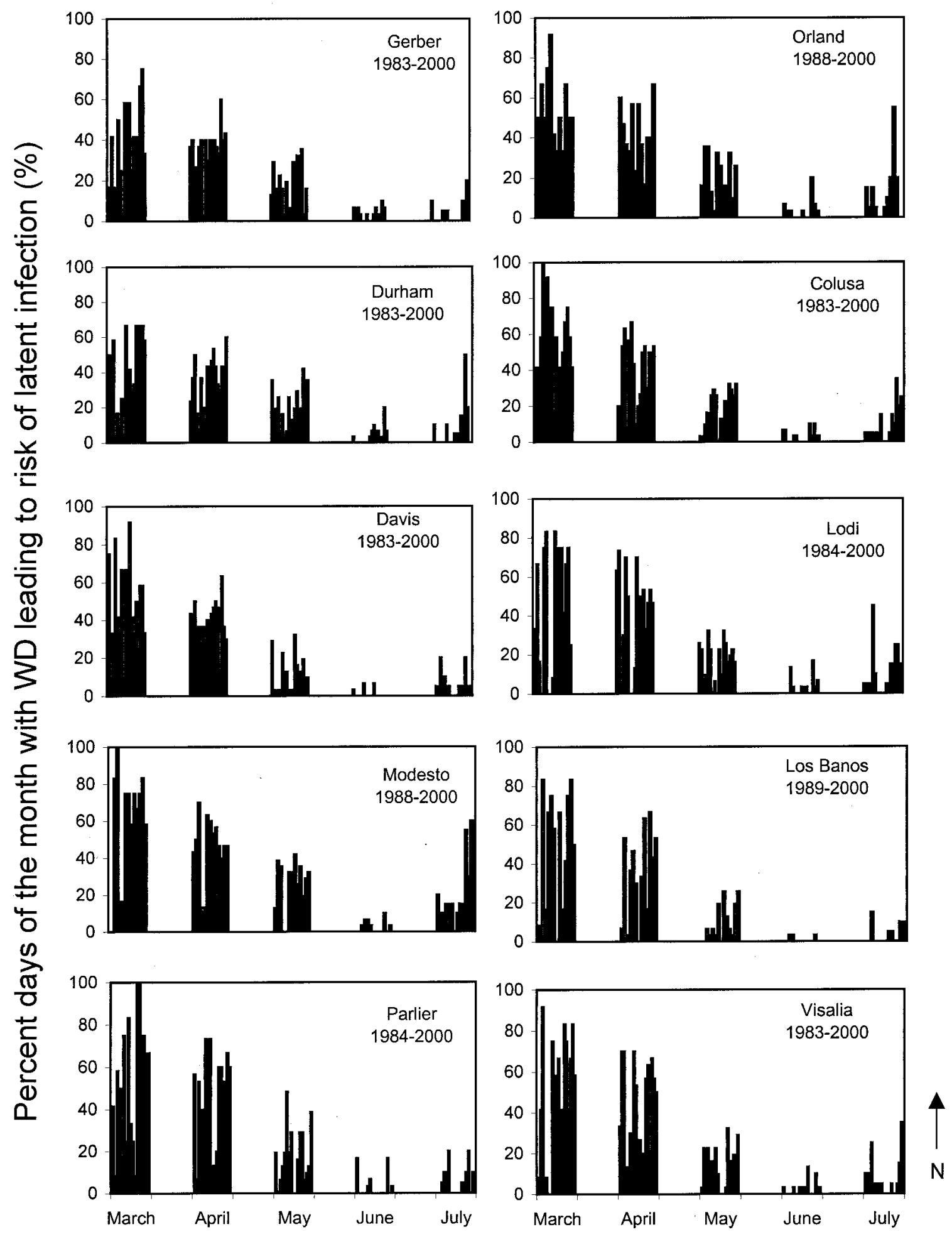

\section{Month of year}

Fig. 4. Distributions of percent days of the month with wetness duration (WD) leading to low risk of latent infection by Monilinia fructicola for each month during the prune-growing season. Multi-year weather data were used in each graph for each prune-growing location of California. Each bar represents a year. The graphs are for the low inoculum potential conditions. 
calculated from each location's historical weather data using the approach described previously. These WD data were used to calculate the $\mathrm{ND}_{L}, \mathrm{ND}_{M}$, and $\mathrm{ND}_{H}$, respectively, for each month from 15 March to 20 July. These ND data were then used to compare the monthly mean threshold $\mathrm{ND}_{L}, \mathrm{ND}_{M}$, and $\mathrm{ND}_{H}$ values for the corresponding locations obtained from the approaches described previously. For each month, when ND was less than the corresponding threshold ND value, no corresponding level of risk of latent infection was estimated. Otherwise, the risk level of latent infection was determined. The overall estimations of risk level were then used to compare the observed incidence of fruit brown rot for each experiment.

Evaluation 2 used the experimental data obtained from orchards in Glenn and Butte counties in California. Similar experiments were conducted from 1994 though 1996 in the same orchards. The objective of the experiments was to study the ecology and epidemiology of prune brown rot and new control strategies $(17,19$, 21). The data used in the evaluation were the means of incidence of fruit brown rot with non-fungicide treatment, noninoculation (naturally infected) control $(17,19,21)$. The same method in comparisons between predicted risk and observed disease incidences was used as evaluation 1.

\section{RESULTS}

Table 1 lists the results of exponential regressions between ILI and WD for bloom and fruit developmental stages combined with three IC. No correlation was determined when IC $=1,000$ conidia

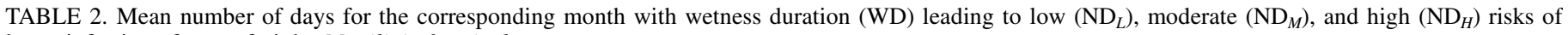
latent infection of prune fruit by Monilinia fructicola ${ }^{\mathrm{a}}$

\begin{tabular}{|c|c|c|c|c|c|c|c|}
\hline \multirow[b]{2}{*}{ Location } & \multirow[b]{2}{*}{ Month } & \multicolumn{3}{|c|}{$\mathrm{IP}_{L}$} & \multicolumn{3}{|c|}{$\mathrm{IP}_{H}$} \\
\hline & & Low risk $\left(\mathrm{ND}_{L}\right)$ & Moderate risk $\left(\mathrm{ND}_{M}\right)$ & High risk $\left(\mathrm{ND}_{H}\right)$ & Low risk $\left(\mathrm{ND}_{L}\right)$ & Moderate risk $\left(\mathrm{ND}_{M}\right)$ & High risk $\left(\mathrm{ND}_{H}\right)$ \\
\hline \multirow{5}{*}{$\begin{array}{l}\text { Gerber } \\
\text { (Tehama County) }\end{array}$} & March & 6.3 & 0.8 & 0.3 & 3.2 & 3.3 & 3.5 \\
\hline & April & 11 & 3.4 & 0.7 & 1.6 & 8.4 & 6.1 \\
\hline & May & 5.5 & 3 & 0.7 & 4.4 & 5 & 2.2 \\
\hline & June & 1 & 0.2 & 0 & 2.6 & 0 & 0.2 \\
\hline & July & 0.7 & 0 & 0 & 0.7 & 0.3 & 0.2 \\
\hline \multirow{5}{*}{$\begin{array}{l}\text { Orland } \\
\text { (Glenn County) }\end{array}$} & March & 8.8 & 1.1 & 0 & 3.8 & 4.8 & 3.8 \\
\hline & April & 12.8 & 4.6 & 0.6 & 1.6 & 10 & 8 \\
\hline & May & 6.7 & 2.3 & 0.7 & 8.1 & 4.2 & 1.8 \\
\hline & June & 1.2 & 0.6 & 0 & 3.2 & 0.1 & 0.6 \\
\hline & July & 2.5 & 0.3 & 0.4 & 2.2 & 1.3 & 0.8 \\
\hline \multirow{5}{*}{$\begin{array}{l}\text { Durham } \\
\text { (Butte County) }\end{array}$} & March & 6 & 0.9 & 0.1 & 3.1 & 3.8 & 2.9 \\
\hline & April & 10.6 & 3.1 & 1.2 & 1.6 & 8.3 & 5.8 \\
\hline & May & 6 & 1.6 & 0.6 & 5.8 & 3.8 & 1.4 \\
\hline & June & 1 & 0.2 & 0 & 2.2 & 0.2 & 0.2 \\
\hline & July & 1.4 & 0 & 0 & 1.8 & 0.6 & 0 \\
\hline \multirow{5}{*}{$\begin{array}{l}\text { Colusa } \\
\text { (Colusa County) }\end{array}$} & March & 8.9 & 0.7 & 0 & 2.8 & 5.3 & 3.2 \\
\hline & April & 11.9 & 4 & 0.2 & 1.1 & 8.9 & 6.5 \\
\hline & May & 5.5 & 2.2 & 1 & 5.5 & 4.1 & 0.9 \\
\hline & June & 0.9 & 0.2 & 0 & 3.4 & 0.2 & 0.2 \\
\hline & July & 1.8 & 0.1 & 0.8 & 1.6 & 1 & 0.8 \\
\hline \multirow{5}{*}{$\begin{array}{l}\text { Davis } \\
\text { (Yolo County) }\end{array}$} & March & 7.3 & 0.9 & 0 & 2.8 & 3.7 & 3.6 \\
\hline & April & 10.6 & 2.7 & 0.6 & 1.4 & 8.2 & 5 \\
\hline & May & 3.3 & 1.6 & 0.7 & 4.3 & 2.6 & 1.5 \\
\hline & June & 0.3 & 0.2 & 0 & 1.9 & 0.1 & 0.1 \\
\hline & July & 1.1 & 0.1 & 0.1 & 0.6 & 0.5 & 0.2 \\
\hline \multirow{5}{*}{$\begin{array}{l}\text { Lodi } \\
\text { (San Joaquin County) }\end{array}$} & March & 7.7 & 0.8 & 0 & 2.9 & 4.6 & 3.4 \\
\hline & April & 13.2 & 3.3 & 0.4 & 1.4 & 10.4 & 6.2 \\
\hline & May & 5.6 & 1.7 & 0.7 & 6.3 & 3.9 & 1.6 \\
\hline & June & 0.9 & 0.2 & 0 & 3.9 & 0.2 & 0.1 \\
\hline & July & 2.2 & 0 & 0 & 1.1 & 1.7 & 0.1 \\
\hline \multirow{5}{*}{$\begin{array}{l}\text { Modesto } \\
\text { (Stanislaus County) }\end{array}$} & March & 9.6 & 0.6 & 0 & 2.4 & 4.8 & 4.8 \\
\hline & April & 13.9 & 5 & 0.2 & 1.1 & 10.9 & 7.8 \\
\hline & May & 8 & 2.1 & 0.7 & 9.4 & 4.9 & 1.9 \\
\hline & June & 0.8 & 2 & 0 & 5.6 & 0.9 & 1.2 \\
\hline & July & 4.1 & 0 & 0 & 3.4 & 2.3 & 0.2 \\
\hline \multirow{5}{*}{$\begin{array}{l}\text { Los Banos } \\
\text { (Merced County) }\end{array}$} & March & 8.6 & 1 & 0 & 4 & 4.7 & 3.6 \\
\hline & April & 11.3 & 1.7 & 0.4 & 1.5 & 9.4 & 3.6 \\
\hline & May & 3.4 & 1.1 & 1.8 & 3.3 & 3 & 1.9 \\
\hline & June & 0.2 & 0.4 & 0 & 0.5 & 0.2 & 0.2 \\
\hline & July & 0.8 & 0 & 0 & 0.8 & 0.4 & 0 \\
\hline \multirow{5}{*}{$\begin{array}{l}\text { Parlier } \\
\text { (Fresno County) }\end{array}$} & March & 8.5 & 0.5 & 0 & 3.7 & 5.1 & 2.8 \\
\hline & April & 14 & 2.5 & 0.2 & 1.9 & 11 & 4.4 \\
\hline & May & 5.4 & 0.7 & 0.3 & 4.7 & 3.6 & 0.7 \\
\hline & June & 0.8 & 0.2 & 0 & 1.5 & 0.1 & 0.2 \\
\hline & July & 1.1 & 0.1 & 0 & 1.6 & 0.3 & 0.1 \\
\hline \multirow{5}{*}{$\begin{array}{l}\text { Visalia } \\
\text { (Tulare County) }\end{array}$} & March & 7.6 & 0.3 & 0 & 3 & 5 & 2.1 \\
\hline & April & 12.1 & 2.3 & 0.2 & 1.8 & 10.6 & 3.3 \\
\hline & May & 4.1 & 0.6 & 0.4 & 3.2 & 2.9 & 0.8 \\
\hline & June & 0.7 & 1.1 & 0 & 1.3 & 0.2 & 1.1 \\
\hline & July & 1.3 & 0 & 0.1 & 1.5 & 0.4 & 0.2 \\
\hline
\end{tabular}

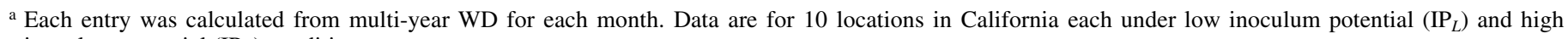
inoculum potential $\left(\mathrm{IP}_{H}\right)$ conditions. 
per $\mathrm{ml}$ at the bloom, embryo growth, and before first harvest stages (Table 1). The regressions for other ICs and developmental stages were significant at $P<0.05$ (Table 1 ).

The curves of WD leading to different risk levels of latent infection by $M$. fructicola over the growing season were produced for the $\mathrm{IP}_{L}$ and $\mathrm{IP}_{H}$ conditions (Fig. 3). Basically, the seasonal patterns of WD leading to different risk levels of latent infection were related to the seasonal patterns of bloom and fruit susceptibility to latent infection (13). Longer WD is required at the resistant stage than at the susceptible stage to induce similar levels of latent infection (Fig. 3). Along with increased susceptibility to latent infection from the bloom to the pit hardening stage (13), the WD leading to different risk levels of latent infection decreased (Fig. 3). After the pit hardening stage, fruit susceptibility to latent
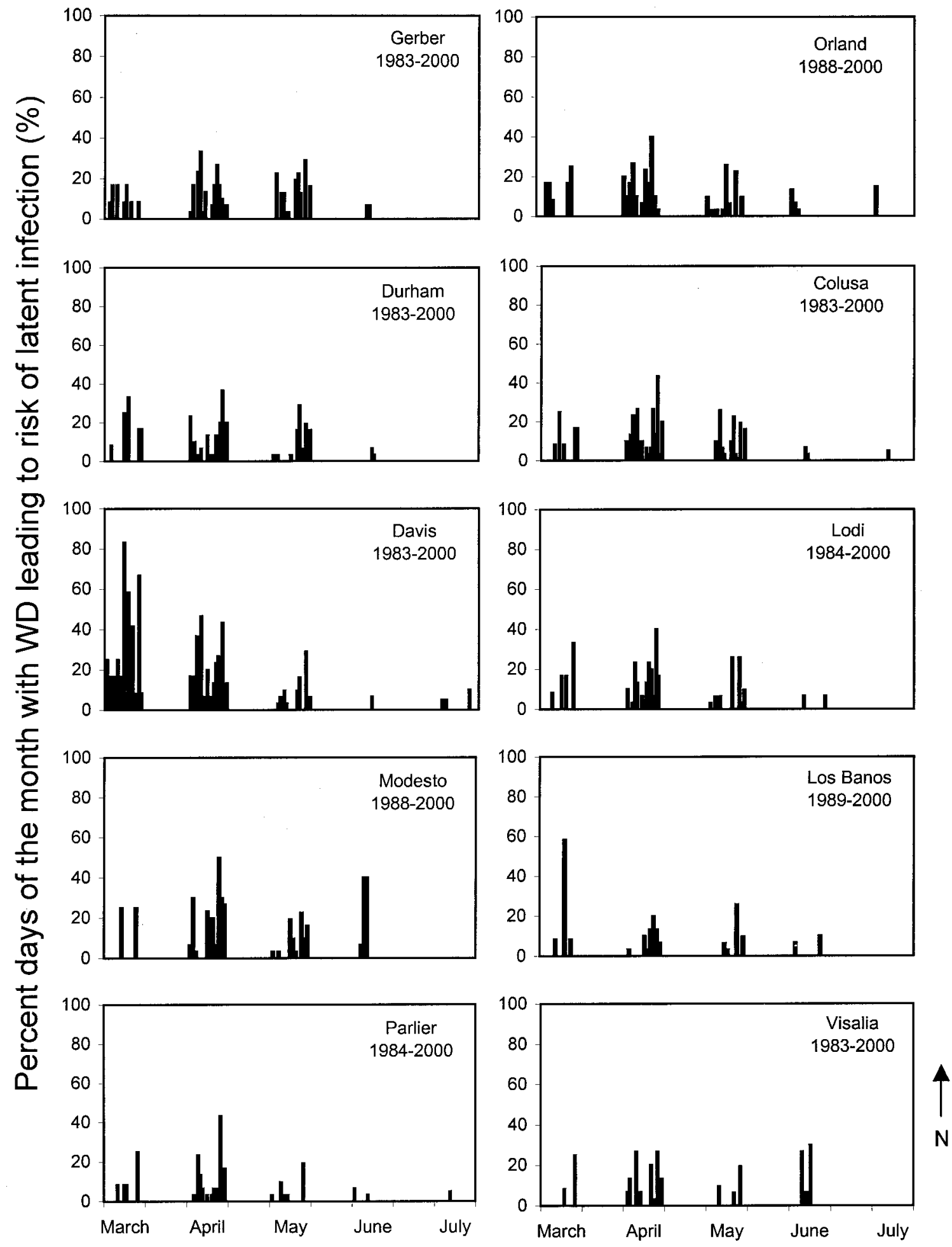

\section{Month of year}

Fig. 5. Distributions of percent days of month with wetness duration (WD) leading to the moderate risk of latent infection by Monilinia fruicticola for each month during the prune-growing season. Each bar represents a year. The graphs are for the low inoculum potential conditions. 
infection dramatically decreased until embryo growth (13), and the WD leading to risk of latent infection increased (Fig. 3A and B). After mid-June, the fruit susceptibility to latent infection increased again (13), and WD leading to risk of latent infection decreased (Fig. 3). However, the WDs decrease slower under the $\mathrm{IP}_{L}$ (Fig. 3A) than under the $\mathrm{IP}_{H}$ (Fig. 3B) condition.

Under the $\mathrm{IP}_{L}$ condition, distributions of the percent days with WD leading to low risk of latent infection $\left(\mathrm{P}_{L}\right)$ in March and April were similar among the 10 locations (Fig. 4). The $\mathrm{P}_{L}$ decreased in May at all locations. Only a few years with very low $\mathrm{P}_{L}$ occurred in June, but the distributions increased in July. The means of $\mathrm{ND}_{L}$ ranged from 6 to 9.6 days in March and from 10.6 to 14 days in April (Table 2). In May, means of $\mathrm{ND}_{L}$ ranged from 3.3 to 8 days (Table 2), and the $90 \%$ cumulative probability of $\mathrm{P}_{L}\left(\mathrm{CP}_{L 90}\right)$ ranged from 22 to $37 \%$ (6.8 to 11.5 days in May) (data not shown). However, the means of $\mathrm{ND}_{L}$ in June ranged from 0.2 to 1.2 days (Table 2), and $\mathrm{CP}_{L 90}$ values ranged from 3.6 to $9.3 \%$ (1 to 2.8 days in June) (data not shown).

Compared with the number of days associated with low risk of latent infection, fewer days with WD leading to moderate risk of latent infection occurred (Table 2). However, the distributions of $\mathrm{P}_{M}$ were different among locations (Fig. 5). In general, there were more years with risky WD in northern than in southern California, and some locations, such as Gerber, Orland, and Colusa in northern Sacramento Valley, showed similar $\mathrm{P}_{M}$ in May to those in March and April (Fig. 5). Two locations in southern San Joaquin Valley, Parlier and Visalia, showed fewer years with $\mathrm{P}_{M}$ compared with the other locations (Fig. 5). Therefore, there were more chances of moderate risk of latent infection in northern than in southern California. These risky WDs mostly occurred in April, and the mean $\mathrm{ND}_{M}$ were comparatively less than the mean $\mathrm{ND}_{L}$ (Table 2). The means of $\mathrm{ND}_{M}$ ranged from 0.6 to 5 days at the most susceptible stage (April to May), and the maximum $\mathrm{CP}_{M 90}$ was about $30 \%$ (approximately 9 days) (data not shown). The probability of moderate risk of latent infection late in the season was very low.

Under the $\mathrm{IP}_{L}$ condition, a few days with WD leading to high risk of latent infection $\left(\mathrm{ND}_{H}\right)$ were observed in a few years (Table 2). The mean $\mathrm{ND}_{H}$ ranged only from 0 to 1.8 days over the season (Table 2), and the most $\mathrm{CP}_{H 90}$ values were below $5 \%$ (data not shown). This fact indicates very low probability of high risk of latent infection under the $\mathrm{IP}_{L}$ condition.

Comparatively, under the $\mathrm{IP}_{H}$ condition, there were more chances that WD leading to low risk of latent infection occurred in May than in April (data not shown). For the locations in Sacramento Valley, even in June, there were still quite a few years that showed WD leading to low risk of latent infection (data not shown). The mean $\mathrm{ND}_{L}$ ranged from 3.2 to 9.4 days in May and from 0.5 to 5.6 days in June (Table 2). The maximum $\mathrm{CP}_{L 90}$ was about $47 \%$ in May and $29 \%$ in June. Therefore, the chance of low risk of latent infection was higher under the $\mathrm{IP}_{H}$ than under the $\mathrm{IP}_{L}$ condition, especially at the resistant stage (Table 2). The means of $\mathrm{ND}_{M}$ ranged from 0 to 0.9 days (Table 2), and $\mathrm{CP}_{M 90}$ values ranged from 0 to $7 \%$ (data not shown). This fact indicates a low probability of moderate risk level. The means of $\mathrm{ND}_{H}$ were greater in March and April than in June and July (Table 2). In May, the means of $\mathrm{ND}_{H}$ ranged from 0.7 to 2.2 days (Table 2) and the $\mathrm{CP}_{H 90}$ values ranged from 4 to $17 \%$ (data not shown). Therefore, the probability of high risk of latent infection under $\mathrm{IP}_{H}$ condition was relatively higher at the susceptible stages than at the resistant stages.

In evaluation 1, predicted overall risk levels of latent infection were consistent with observed incidences of brown rot of fruit. Two orchards showed the disease incidence within the moderate
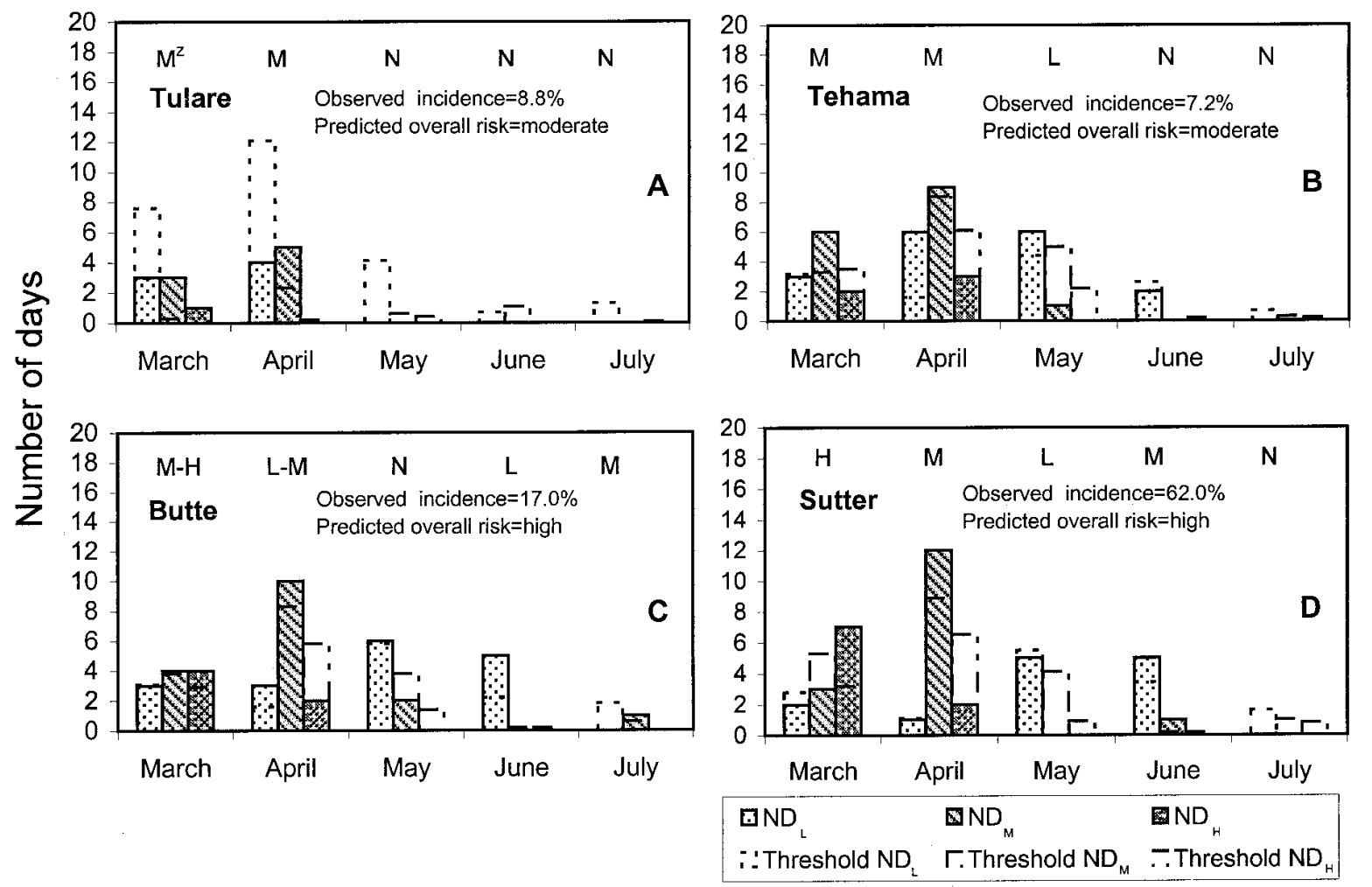

\section{Month of year}

Fig. 6. The predicted risk levels of latent infection of prune by Monilinia fructicola based on number of days with wetness duration leading to low $\left(\mathrm{ND}_{L}\right)$, moderate $\left(\mathrm{ND}_{M}\right)$, and high $\left(\mathrm{ND}_{H}\right)$ risk levels of latent infection for the corresponding month, in comparison with the corresponding threshold values. The overall risk levels are compared with the observed incidences of brown rot of prune fruit. The observations were from experiments in four locations in California in 1993 (20). ${ }^{\mathrm{z}}$ The predicted risk levels of latent infection: $\mathrm{N}=$ no risk, $\mathrm{L}=$ low risk, $\mathrm{M}=$ moderate risk, and $\mathrm{H}=$ high risk. 
risk level of latent infection, $8.8 \%$ at Tulare (Fig. 6A) and $7.2 \%$ at Tehama (Fig. 6B), and the other two orchards showed the disease incidence within the high risk level of latent infection, $17 \%$ at Butte (Fig. 6C) and 62\% at Sutter (Fig. 6D). For the Tulare orchard in March (Fig. 6A), the $\mathrm{ND}_{L}$ was 3 days, less than the mean threshold $\mathrm{ND}_{L}$ (7.6 days) (Table 2, for Visalia), but $\mathrm{ND}_{M}$ was 3 days, greater than the mean threshold $\mathrm{ND}_{M}$ (0.3 days). There was also a day with WD leading to a high risk of latent infection (Fig. 6A). Therefore, a moderate risk of latent infection was predicted for this month. In April, the $\mathrm{ND}_{M}$ was 5 days, greater than the mean threshold $\mathrm{ND}_{M}$ at 2.3 days (Table 2, for Visalia), and neither $\mathrm{ND}_{L}$ nor $\mathrm{ND}_{H}$ was greater than the corresponding threshold $\mathrm{ND}_{L}$ or $\mathrm{ND}_{H}$ values, respectively. Thus, a moderate risk of latent infection was predicted in April (Fig. 6A). A similar situation was observed in the Gerber orchard (Tehama County), and the threshold ND values in Table 2 for Gerber were used in the comparisons. A moderate risk of latent infection was predicted in March and April, and a low risk of latent infection was predicted in May (Fig. 6B). An overall moderate risk of latent infection was predicted (Fig. 6B), and the observed average fruit brown rot $(7.2 \%)$ was within the range of moderate risk level of latent infection.

Different from the above situations, for the orchard in Butte County, both $\mathrm{ND}_{M}$ (4 days) and $\mathrm{ND}_{H}$ (4 days) in March (Fig. 6C) were greater than the respective mean threshold $\mathrm{ND}_{M}$ (3.8 days) and $\mathrm{ND}_{H}$ (2.9 days) (Table 2, for Durham). In April, both $\mathrm{ND}_{L}$ (3 days) and $\mathrm{ND}_{M}$ (10 days) were greater than the respective mean threshold $\mathrm{ND}_{L}$ (1.6 days) and $\mathrm{ND}_{M}$ (8.3 days). Therefore, a moderate to high risk of latent infection was predicted in March, and a low to moderate risk of latent infection was predicted in April (Fig. 6C). A low risk of latent infection was predicted in June, and a moderate risk of latent infection was predicted in July. By summarizing the situations over the growing season, a high risk of latent infection was predicted, and the observed incidence of fruit brown rot was $17 \%$ (Fig. 6C). The information for Colusa County in Table 2 was used in the evaluation process for the Sutter orchard, because this orchard was close to the Colusa weather station (about $40 \mathrm{~km}$ ). A high risk of latent infection was predicted in March, and a moderate risk of latent infection was predicted in April. A moderate risk of latent infection was also predicted in June. After considering the situations over the whole season, a high risk of latent infection was predicted and the observed incidence of fruit brown rot was 62\% (Fig. 6D).

In evaluation 2, the two orchards in Glenn and Butte counties were considered under the $\mathrm{IP}_{H}$ condition, because apothecia were abundant under the trees in spring (T. J. Michailides, unpublished data). In the Glenn orchard, the incidence of fruit brown rot was $28.2 \%$ in 1994 . No risk was predicted in March, but a moderate risk was predicted in April. In May, there was 5 and 3 days that WD leading to a moderate and a high risk of latent infection occurred (Fig. 7A), greater than the mean threshold $\mathrm{ND}_{M}$ (4.2 days) and $\mathrm{ND}_{H}$ (1.8 days), respectively. This weather pattern occurring at the susceptible stages (13) might be a causal reason of the severe brown rot. In 1995, a low risk was predicted in March, June, and July, and a high risk was predicted in April and May. The observed incidence of fruit brown rot was $21.3 \%$ (Fig. 7B). In 1996, more severe fruit brown rot was observed (incidence $=$ $44.9 \%$ ). A moderate risk of latent infection was predicted in March, a high risk of latent infection was predicted in April, and a low risk of latent infection was predicted in July (Fig. 7C).

For the orchard in Butte County, the information about risk criteria for Durham (Table 2, for Butte County) was used in the evaluation process. The observed incidence of fruit brown rot was $11.8 \%$ in 1994 (Fig. 7D). A low risk of latent infection was predicted in March, and a high risk of latent infection was predicted in May. In 1995, although no risky day occurred in March, there was 15 days that WD leading to a moderate risk of latent infection occurred in April (Fig. 7E). A high risk of latent infection was encountered in May, because $\mathrm{ND}_{L}, \mathrm{ND}_{M}$, and $\mathrm{ND}_{H}$ were all greater than the corresponding threshold values (Fig. 7E). A low risk of latent infection was also predicted in June and July (Fig. 7E). The pattern of continuous risky weather that occurred in April and May might have led to a high incidence of fruit brown rot $(51.3 \%)$. The situations in 1996 were slightly different. A moderate risk was predicted in March (Fig. 7F), and the weather patterns were more favorable for infection in April than in March (Fig. 7F). A low risk of latent infection was predicted in May and July (Fig. 7F). Occurrence of continuous risky weather patterns in March and April might have led to the high incidence of fruit brown rot at $48.9 \%$.

\section{DISCUSSION}

This study determined the seasonal patterns of WD leading to different risk levels of latent infection of prune by $M$. fructicola. Because the data used in this study were from field experiments at different locations in California, the curves of risky WD over the growing season could be used as reference to guide growers in decision making for disease management. To simplify the use of the results, the $\mathrm{IP}_{L}$ could roughly refer to the orchards where brown rot infrequently occurred and apothecia were infrequently found. The $\mathrm{IP}_{H}$ could refer to the orchards where abundant apothecia were frequently found in the spring and brown rot frequently occurred. Thus, the curves in Figure $3 \mathrm{~A}$ could be used under the $\mathrm{IP}_{L}$ condition to determine if either a current or predicted WD of a certain day or a period of days may relate to what risk level of latent infection. When the WD is below the low-risk curve, fungicide sprays may not be necessary if the grower could sustain a risk of $1 \%$ latent infection. However, if the WD is located between the low- and moderate-risk curves on a certain day, a decision for disease control may depend entirely on the grower's attitude toward risk. When the predicted WD is between the moderate- and high-risk curves, most growers may decide to spray because the predicted ILI levels will be at 5 to $10 \%$. When the predicted WD is above the high-risk curve, a fungicide spray will be highly recommended.

A similar approach could be applied for orchards under the $\mathrm{IP}_{H}$ condition (Fig. 3B). Because the data in relation to low-risk curve in March and April are not available from the data analysis, the risky WD in these 2 months could not be determined (Fig. 3B). The high-risk curve is higher and sharper at the end of May under the $\mathrm{IP}_{H}$ than under the $\mathrm{IP}_{L}$ condition. This fact implies that during the period of most resistance to latent infection, even high IP does not necessarily relate to high risk of latent infection. In other words, much longer WD may be needed to induce a high ILI.

Obviously, not all fruit with latent infection could develop brown rot prior to harvest. Therefore, the ILI may be greater than the observed incidence of fruit brown rot. The conditions under which latent infection could lead to disease appearance are complex. These conditions may include high latent infection levels early and late in the growing season and high humidity during the period of disease appearance. This information is important to determine when a fungicide application is most efficient.

This study not only produced a tool as a reference to decision making for disease control, but also provided information on probabilities of historical occurrence of risk of latent infections. The information is useful especially in decision making when the predicted or observed WDs are between the low- and high-risk curves. In making decisions for fungicide spray, growers may need to know a similar historical weather situation and how high the probability of a similar weather and disease situation was. If the current situation leads to higher risk of latent infection than an average situation, a fungicide spray is necessary. For example, in an orchard at Gerber, located in northern Sacramento Valley, if the predicted WD is $10 \mathrm{~h}$ on 10 May, the WD leads to a moderate risk of latent infection (Fig. 3A) under $\mathrm{IP}_{L}$. The average percentage of days with such WD condition that occurred historically in this month is $10 \%$ (3 days), 
and the $\mathrm{P}$ with $90 \%$ cumulative probability $\left(\mathrm{CP}_{M 90}\right)$ is $21 \%$ (6.3 days). According to the above estimation, the grower may decide to spray because in this period prunes are at a very susceptible stage (13), and only an average of 3 days of this month with the same situation occurred historically. If the disease infrequently occurred in the past in the specific orchard, the current situation may lead to a latent infection level that needs to be reduced. However, the grower may decide not to spray to sustain a certain risk because such risky WD may probably occur only 1 day in this month in the future. In addition to Table 2, other tables, containing the information such as $\mathrm{P}$ and $\mathrm{CP}_{90}$, produced by this study, are useful to guide disease control for a specific location, and can be implemented in a decision support system. A grower can make a better decision for disease management by using a precise weather forecast combined with our risk analysis tools.

Comparing observed incidence of fruit brown rot with predicted risk level of latent infection provided some clues about the impor- tance of seasonal weather patterns in risks of latent infection. This study demonstrated that the risky WD during bloom season was highly related to the incidence of fruit brown rot. For example, higher incidences of fruit brown rot were observed in the Butte and Sutter orchards than in the Tulare and Tehama orchards (Fig. 6), and the predicted risk levels of latent infection in March were consistent with the observations. The weather patterns in May and June might also be important in disease prediction and control because fruit susceptibility to latent infection could change dramatically from susceptible to resistant during this period (13). For example, the higher incidence of fruit brown rot observed in the Sutter orchard than in the other orchards may be due to the fact that only the Sutter orchard showed higher risk levels of latent infection (low risk in May and moderate risk in June) than the other three orchards (Fig. 6).

The evaluation 2 provided information on how weather patterns were related to high levels of incidence of fruit brown rot. By
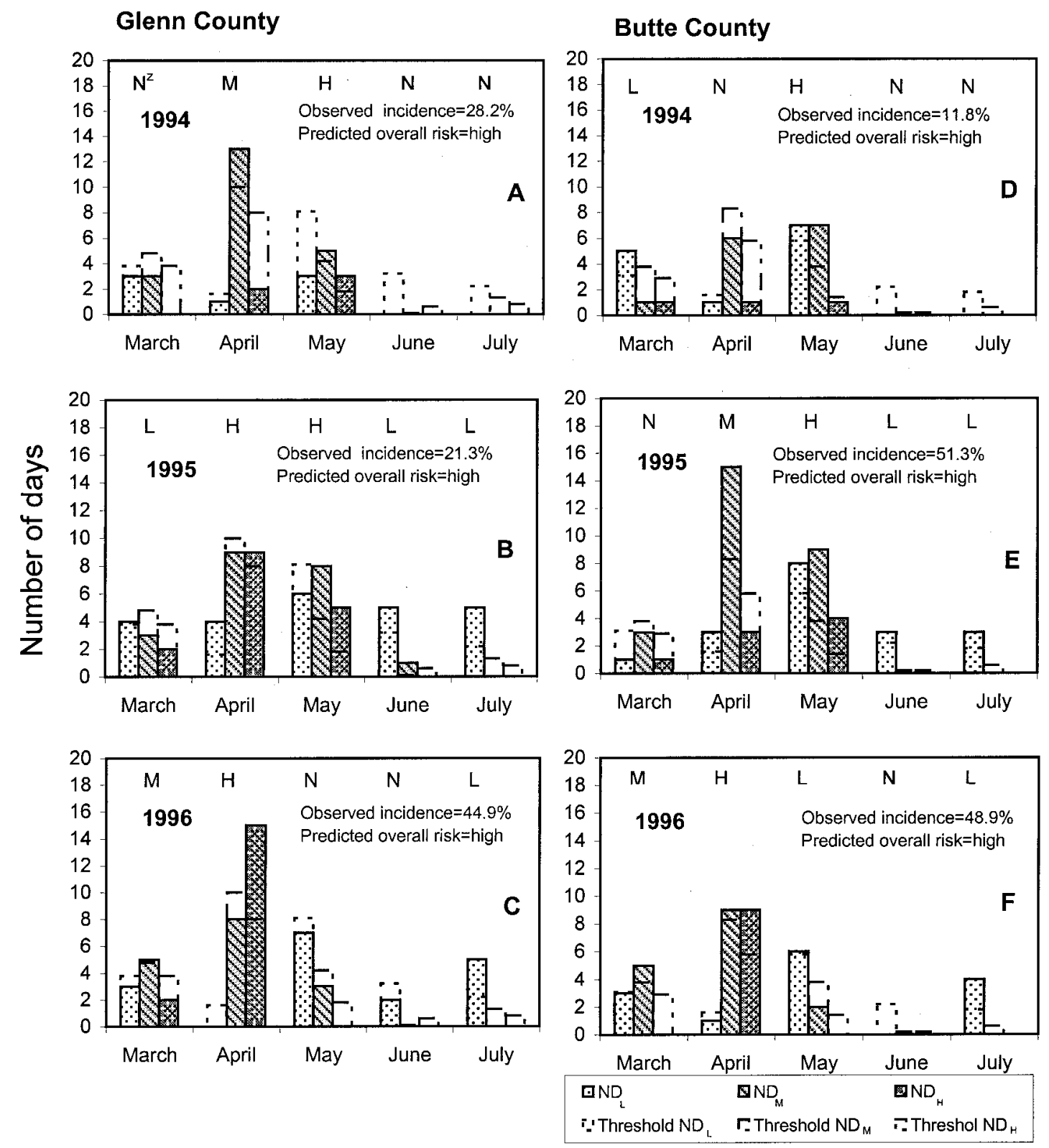

\section{Month of year}

Fig. 7. The predicted risk levels of latent infection of prune fruit by Monilinia fructicola based on number of days with wetness duration leading to low (ND $)_{L}$, moderate $\left(\mathrm{ND}_{M}\right)$, and high $\left(\mathrm{ND}_{H}\right)$ risk levels of latent infection for the corresponding month, in comparison with the corresponding threshold values. The overall risk levels are compared with the observed incidences of brown rot of prune fruit. The observations were form experiments in two locations in California each for 3 years $(17,19,21){ }^{\mathrm{z}}$ The predicted risk levels of latent infection: $\mathrm{N}=$ no risk, $\mathrm{L}=$ low risk, $\mathrm{M}=$ moderate risk, and $\mathrm{H}=$ high risk. 
comparing weather conditions with observed disease incidence, we conclude that a continuous weather pattern favorable to disease development occurring early in the growing season may bring highly severe fruit brown rot. More days of favorable weather from March to May can enhance latent infections that may lead to symptoms and sporulation late in the season. The results of the evaluation 2 also provided an outline on possible combinations of risky weather patterns and subsequent levels of disease incidence. For example, if $50 \%$ of the days in April or May showed WD leading to a high risk of latent infection, there would be a high possibility that severe fruit brown rot may occur late in the season. A similar level of disease intensity may also occur when some days (e.g., $30 \%$ of the days of the month) of highly favorable weather occur in both March and April or in both April and May. How different weather patterns at different fruit developmental stages could lead to different disease levels is important information for disease prediction.

Decision making for disease control needs the information on the effectiveness of fungicides, especially when a fungicide spray is applied at bloom or early in the season. For example, when WD conditions are related to a high risk of latent infection in a period that is within the fungicide residual effect period, an additional fungicide spray may not be necessary. Therefore, a study on fungicide effectiveness, together with the risk assessment for disease control, is an intensive research topic that may benefit prune production in California.

The seasonal pattern of bloom and fruit susceptibility to latent infection by $M$. fructicola combined with the seasonal patterns of risky WD conditions outline disease control strategies. In California, fungicide sprays at the green tip stage to control blossom blight of prunes is commercially recommended. However, based on our previous study (13), the susceptibility to infection by $M$. fructicola increased after fruit set, especially when fruit are very young. This situation may last from mid-April to mid-May, and weather conditions during this period are usually flexible in prune-growing areas in California. Infections early in the season might have more chances of disease appearance before fruit become mature than those late in the season. Therefore, the period from April to May is critical in disease control for which the risk information and decision support are helpful. After mid-May, fruit resistance to latent infection increases, and dry weather with much less rain occurs until harvest in these areas. The risk of latent infection during this period decreases, and in most cases, a fungicide spray to control disease may not be recommended. Because information about the latest period of latent infection, which could lead to the disease appearance (sporulation on fruit) before harvest, is not yet available, the importance in disease control late in the growing season is still understudied. Even though fruit susceptibility to latent infection increases in July (13), disease control may not be necessary. But, spraying trees just before the fruit-to-fruit contact stages of prune fruit, which favors brown rot infection (15), may reduce brown rot incidence at harvest. Although a risky WD may be encountered to lead latent infections during this period, disease may not appear before harvest. Moreover, postharvest brown rot may not be important in prunes, especially since prunes are dehydrated immediately after harvest in the majority of California prune production. However, decision making for disease control in this late period should rely on intensive studies to understand the disease development process late in the season.

Temperature was one factor affecting latent infection of fruit by $M$. fructicola that was determined in our previous studies $(12,13)$. Temperatures ranged from 10 to $35^{\circ} \mathrm{C}$ throughout the season of the experiments. Because this study involved a risk analysis of latent infection by considering $10 \%$ ILI as the highest risk level of latent infection, a temperature range, relating to less or equal to $10 \%$ of ILI calculated from the previous study, was determined. The historical temperatures during the period of 6:00 p.m. to 10:00 a.m. were used, and the temperature ranges encountered in the experiments, relating to less or equal to $10 \%$ of ILI, were within the historical temperature ranges. Therefore, the curves of risky WD could represent the normal temperature situations in fields.

The IP in different orchards is important information for disease control. Obviously, the IP in an orchard may change during the growing season because it is affected by weather, cultural practices, pathogen survival under different conditions, and other factors. Simply classifying IP into low and high levels as it was done in this study may not represent all possible IP situations. The sensitivity of IP in decision making, namely how detailed levels of IP are needed, is still unknown. Possible methods to determine the IP in orchards over the growing season are under investigation.

In addition to the previous study on risk analysis for blossom blight of prune caused by $M$. fructicola (14), this study continued to determine the risk of latent infection during the bloom and fruit developmental stages. Combination of these two series of studies provides an outlook of risk of brown rot of prune over the growing season. Because the two phases of brown rot disease have been thus far considered in risk analysis, the information could now be available in developing a decision support system and integrated pest management strategies with application over a larger area. These approaches and results might also be useful in studies of brown rot disease of other stone fruit that are stored after harvest, with an importance in postharvest brown rot.

\section{ACKNOWLEDGMENTS}

This study was supported by the California Dried Plum Board. We thank D. Morgan, Kearney Agricultural Center, for technical assistance in previous experiments, data of which were used for the evaluation of risk analysis approaches, and K. Lynn, Kearney Agricultural Center, for help in producing the GIS map.

\section{LITERATURE CITED}

1. Biggs, A. R., and Northover, J. 1985. Inoculum sources for Monilinia fructicola in Ontario peach orchards. Can. J. Plant Pathol. 7:302-307.

2. Biggs, A. R., and Northover, J. 1988. Early and late-season susceptibility of peach fruits to Monilinia fructicola. Plant Dis. 72:1070-1074.

3. Biggs, A. R., and Northover, J. 1988. Influence of temperature and wetness duration on infection of peach and sweet cherry fruits by Monilinia fructicola. Phytopathology 78:1352-1356.

4. Byrde, R. J. W., and Willetts, H. J. 1977. The Brown Rot Fungi of Fruit: Their Biology and Control. Pergamon Press, Oxford.

5. Corbin, J. B. 1963. Factors determining the length of the incubation period of Monilinia fructicola (Wint.) Honey in fruits of Prunus spp. Aust. J. Agric. Res. 14:51-60.

6. Cruickshank, R. H., and Wade, G. C. 1992. The activation of latent infections of Monilinia fructicola on apricots by volatiles from the ripening fruit. J. Phytopathol. 136:107-112.

7. Emery, K. M., Michailides, T. J., and Scherm, H. 2000. Incidence of latent infection of immature peach fruit by Monilinia fructicola and relationship to brown rot in Georgia. Plant Dis. 84:853-857.

8. Hong, C. X., Holtz, B. A., Morgan, D. P., and Michailides, T. J. 1997. Significance of thinned fruit as a source of the secondary inoculum of Monilinia fructicola in California nectarine orchards. Plant Dis. 81:519524.

9. Jerome, S. M. R. 1958. Brown rot of stone fruits: Latent contamination in relation to spread of the disease. J. Aust. Inst. Agric. Sci. 24:132-140.

10. Kable, P. F. 1965. Air dispersal of spores of Monilinia fructicola in peach orchards. Aust. J. Exp. Agric. Anim. Husb. 5:166-171.

11. Landgraf, F. A., and Zehr, E. 1982. Inoculum sources for Monilinia fructicola in South Carolina peach orchards. Phytopathology 72:185-190.

12. Luo, Y., Ma, Z., and Michailides, T. J. 2001. Analysis of factors affecting latent infection and sporulation of Monilinia fructicola on prune fruit. Plant Dis. 85:999-1003.

13. Luo, Y., and Michailides, T. J. 2001. Factors affecting latent infection of prune fruit by Monilinia fructicola. Phytopathology 91:864-872.

14. Luo, Y., Morgan, D. P., and Michailides, T. J. 2001. Risk analysis of brown rot blossom blight of prune caused by Monilinia fructicola. Phytopathology 91:759-768.

15. Michailides, T. J., and Morgan, D. P. 1997. Influence of fruit-to-fruit contact on the susceptibility of French prune to infection by Monilinia fructicola. Plant Dis. 81:1416-1424.

16. Michailides, T. J., Morgan, D. P., and Felts, D. 2000. Detection and 
significance of symptomless latent infection of Monilinia fructicola in California stone fruit. (Abstr.) Phytopathology 90(suppl.):S48.

17. Michailides, T. J., Morgan, D. P., Felts, D., and Krueger, W. 1996. Ecology and epidemiology of prune brown rot and new control strategies. Pages 109-123 in: Prune Research Report and Index of Prune Research, California Prune Board, Pleasanton.

18. Michailides, T. J., Morgan, D. P., Holtz, P. A., Guo, L., and Hong, C. X. 1995. Cultural and chemical controls of preharvest and postharvest brown rot of peach, nectarines, and plums, and Mucor rot of late varieties of nectarines and plums. Pages 86-101 in: Res. Rep. Calif. Tree Fruit Agreement, Reedley, CA.

19. Michailides, T. J., Morgan, D. P., Holtz, P. A., and Hong, C. X. 1995. Biology, ecology, and epidemiology of Monilinia species, and management of prune brown rot with late-spring and early-summer fungicide sprays. Pages 79-100 in: Prune Research Report and Index of Prune Research, California Prune Board, Pleasanton.

20. Michailides, T. J., Morgan, D. P., and Kölliker, R. 1993. Effects of early and mid summer sprays on the postharvest brown rot of French prune.
Pages 140-157 in: Prune Research Report and Index of Prune Research, California Prune Board, Pleasanton.

21. Michailides, T. J., Morgan, D. P., Sibbett, S. G., and Teviotdale, B. L. 1994. Management of brown rot of French prune by detecting infections in contact surfaces and by early and late summer fungicide applications. Pages 63-86 in: Prune Research Report and Index of Prune Research, California Prune Board, Pleasanton.

22. Phillips, D. J. 1984. Effect of temperature on Monilinia fructicola spores produced on fresh stone fruits. Plant Dis. 68:610-612.

23. Polito, V. S. 1981. Flower and fruit development. Pages 46-52 in: Prune Orchard Management. D. E. Ramos, ed. Univ. Calif. Div. Agric. Sci. Publ. 3269.

24. Sholberg, P. L., Ogawa, J. M., and Manji, B. T. 1981. Diseases of prune blossoms, fruits, and leaves. Pages 121-125 in: Prune Orchard Management. Univ. Calif. Div. Agric. Sci. Publ. 3269.

25. Wade, G. C., and Cruickshank, R. H. 1992. The establishment and structure of latent infections with Monilinia fructicola on apricot. J. Phytopathol. 136:95-106. 